\title{
Editors' Foreword
}

With this issue the JLG begins its $6^{\text {th }}$ year. We believe we have brought significant scholarship in modern dialectology to the web-based publication format that meets many of its technical needs - embedded sound files, the ability to zoom and pan high-quality graphics (in full color), and manipulable data sets. We also believe that our articles have been much more than high-tech; a review of them shows serious concerns with advances in and proposals for historical dialectology, geographical and statistical innovations in mapping and data handling, typological concerns, and full recognition of the warning given by Chambers and Trudgill some years ago: "Dialectology without sociolinguistics at its core is a relic" (Dialectology, 1998, $2^{\text {nd }}$ pbk ed., p. 188).

To date (including 6.1) we have considered (or are considering) seventy-three submissions. Thirty-seven, including two book reviews, have appeared (an acceptance rate only very slightly better than fifty percent), four are being revised, eight are under review, twentyone were not accepted, and three were withdrawn. This relatively high acceptance rate reflects a number of invited (but fully refereed) papers in the first year or two of the journal before its presence was better established. Quality submissions to the journal have reached the level so that four articles (or three and a book review) are now included in each number, and, beginning in 6.2, we will include a submission history for each. Our first special issue will appear in 2018 (6.2), a selection of papers from the conference Spatial Boundaries and Transitions in Language and Interaction: Perspectives from Linguistics and Geography, April 23 - 28, 2017 Monte Verità, Ascona, Switzerland, organized by URPP Language and Space, the University of Zurich.

\section{Introducción}

Con este volumen, la revista JLG comienza su sexto año de andadura. Creemos estar contribuyendo al desarrollo académico de la dialectología moderna llevándola a un formato de publicación en red que satisface la mayoría de sus necesidades técnicas: archivos de sonido integrados, capacidad de acercar y alejar gráficos de alta calidad (a todo color) y bases de datos manipulables. También creemos que nuestros artículos han aportado mucho más que alta tecnología. En ellos se aprecia un serio interés por los avances y propuestas de la dialectología histórica, la innovaciones geográficas y estadísticas en el cartografiado y la manipulación de datos, las cuestiones de tipología y el pleno reconocimiento a la advertencia que Chambers y
We also announce a few changes.

Bill Labov, whose brainchild the journal was and who served as founding Co-editor for its first five years, has resigned that position to become the Senior Editorial Advisor. Bill's belief in the value of dialectology was the driving force in this journal's founding and early years. We were very lucky to have his expertise and interests devoted to the journal during that period and look forward to continuing our association with him, turning to him often for the wisdom he gives to all things variationist.

Our new Co-editor is Francisco Moreno-Fernández, Professor of Hispanic Linguistics at the University of Alcalá de Henares (Spain), Executive Director of the Observatory of the Spanish Language and Hispanic Cultures in the United States at Harvard University, and former Academic Director of the Instituto Cervantes. $\mathrm{He}$ is an expert in varieties of Spanish in both Latin America and Spain, multilingualism, and sociolinguistic theory and application and has directed large, multinational surveys in many areas of the Spanish-speaking world. He brings complementary regional and specialist interests to the journal.

Our Editorial Advisory Board has been updated and is shown on our opening webpage. We thank those who have served, those who will continue to serve, and welcome new specialists. Our thanks also to all our authors and reviewers for our first five years. We look forward to the next five.

We continue to welcome submissions in all areas of linguistic geography, perhaps particularly from those who use advances in geographical interpretations of linguistic diversity and those who treat typological issues.

Trudgill hicieron hace algunos años: "La dialectología sin sociolingüística en su núcleo es una reliquia" (Dialectology, 1998, 2nd ed., p 188).

Hasta la fecha (incluyendo el volumen 6.1) hemos considerado (o estamos considerando) la publicación de 73 propuestas recibidas. De ellas, se han publicado el $37 \%$, incluyendo dos reseñas de libros (con una tasa de aceptación algo mayor del 50\%), 4 están siendo evaluadas, 8 están en proceso de revisión, 21 no fueron aceptadas y 3 fueron retiradas. Este nivel de aceptación, relativamente alto, incluye los artículos invitados durante el primer año (sometidos, eso sí, a una completa evaluación) o dos trabajos concluidos con anterioridad al 
lanzamiento definitivo de la revista. Las propuestas que en este momento llegan a JLG atesoran una calidad suficiente como para incluir en cada número cuatro artículos (o tres artículos y una reseña) y, a partir del volumen 6.2, incluiremos el historial editorial de cada uno de ellos. Nuestro primer número especial aparecerá en 2018 (6.2) y estará conformado por una selección de artículos procedentes del congreso Spatial Boundaries and Transitions in Language and Interaction: Perspectives from Linguistics and Geography ("Fronteras espaciales y transiciones en lengua e interacción: Perspectivas desde la lingüística y la geografía"), celebrado del 23 al 28 de abril de 2017 en Monte Verità, Ascona, Suiza, y organizado por “URPP Language and Space" de la Universidad de Zurich.

Anunciamos también algunos cambios. Bill Labov, promotor de la revista y coeditor fundador durante sus primeros cinco años, ha renunciado a este cargo para convertirse en asesor editorial "senior" de JLG. El convencimiento de Bill sobre el valor de la dialectología fue la fuerza motriz para la fundación y los primeros años de esta revista. Hemos sido afortunados de contar con su experiencia e interés durante todo ese período, y esperamos seguir vinculados a él y recurrir a menudo a su sabiduría sobre asuntos variacionistas.

Nuestro nuevo coeditor es Francisco MorenoFernández, catedrático de Lingüística Hispánica de la

\section{Introdução}

Com este volume, a revista JLG está iniciando o seu sexto ano de publicações. Acreditamos estar contribuindo para o desenvolvimento acadêmico da dialetologia moderna, conduzindo-a a um formato de publicação em rede que satisfaz a maior parte de suas necessidades técnicas: arquivos de som integrados, capacidade de aproximar e distanciar gráficos de alta qualidade (em cores) e bases de dados manipuláveis. Também acreditamos que os artigos publicados nessa revista têm contribuído muito mais que a alta tecnologia. No material produzido na revista tem sido possível apreciar o firme interesse dos pesquisadores pelos avanços e propostas da dialetologia histórica, inovações geográficas e estatísticas na cartografia e na manipulação de dados, questões de tipologia e o pleno reconhecimento da advertência que Chambers e Trudgill fizeram há alguns anos: "A dialetologia que não incorpora a sociolinguística no seu núcleo é uma relíquia "(Dialectology, 1998, 2nd ed., p 188, tradução própria).

Até o presente momento (incluindo o volume 6.1) nós consideramos tivemos a oportunidade de considerar a publicação de 73 propostas recebidas. Dessas propostas, $37 \%$ foram publicadas, incluindo entre essas publicações
Universidad de Alcalá (España), director ejecutivo del Observatorio de la Lengua Española y la Culturas Hispanas en los Estados Unidos en la Universidad de Harvard, y exdirector académico del Instituto Cervantes. Es experto en variedades del español de Latinoamérica y España, en multilingüismo, en sociolingüística teórica y aplicada, y ha dirigido grandes proyectos multinacionales en diversas áreas del mundo hispanohablante. Él aportará a la revista el interés por nuevas regiones y especialidades.

Nuestro Consejo Editorial ha sido renovado, tal y como se muestra en la página web de la revista, por lo que queremos expresar nuestro agradecimiento a todos aquellos que han colaborado con JLG y a aquellos que continuarán haciéndolo, al tiempo que damos la bienvenida a los nuevos expertos colaboradores. Nuestro agradecimiento también a todos los autores y a los que han actuado como revisores durante nuestros primeros cinco años de existencia. Esperamos los próximos cinco con el máximo interés.

Por lo demás, continuaremos aceptando propuestas de todas las áreas de la geografía lingüística, particularmente de aquellas interesadas por los avances en las interpretaciones geográficas de la diversidad lingüística y por las cuestiones tipológicas.

2 resenhas de livros (com uma taxa de aceitação um pouco maior do que 50\%), 4 propostas estão sendo avaliadas, 8 estão em processo de revisão, 21 não foram aceitas e 3 foram retiradas. Este nível de aceitação, que é relativamente alto, incluiu artigos convidados durante o primeiro ano (os quais, como é natural, passaram por uma etapa completa de avaliação) e 2 trabalhos concluídos que foram antes do lançamento definitivo da revista. As propostas que tem chegado à JLG tem qualidade suficiente para que, em cada número da revista, sejam incluídos 4 artigos (ou 3 artigos e 1 resenha) e, a partir do volume 6.2, se passará a incluir o histórico editorial de cada um deles. O primeiro número especial da revista aparecerá a partir deste ano -2018(no volume 6.2); esse volume reunirá uma seleção de artigos procedentes do Congresso Spatial Boundaries and Transitions in Language and Interaction: Perspectives from Linguistics and Geography ("Fronteiras espaciais e transições na língua e interação: perspectivas a partir da linguística e da geografia"), que foi celebrado entre os dias 23 e 28 de abril de 2017 no Monte Verità, Ascona, Suiza, e organizado por "URPP Language and Space" da Universidade de Zurique. 
Nós também queremos anunciar algumas mudanças. Bill Labov, promotor da revista e coeditor fundador da mesma durante seus primeiros 5 anos de existência, renunciou a essa função para assumir o papel de assessor editorial "sênior" da JLG. O convencimento de Bill sobre o valor da dialetologia foi a força motriz para que essa revista fosse fundada e mantida ao longo dos seus primeiros anos. Nós tivemos a sorte de poder contar com a sua experiência e interesse durante todo esse tempo, e esperamos continuar vinculados a ele, recorrendo à sua expertise sobre temas variacionistas.

O nosso novo coeditor é Francisco Moreno-Fernández, Catedrático de Linguística Hispânica da Universidad de Alcalá (Espanha), Diretor executivo do Observatorio de la Lengua Española y la Culturas Hispanas nos Estados Unidos na Universidade de Harvard, e Ex-diretor acadêmico do Instituto Cervantes. Francisco MorenoFernández é especialista na temática das variedades do espanhol da América Latina e da Espanha, em multilinguismo, em sociolinguística teórica e aplicada, tendo dirigido grandes projetos multinacionais em diversas áreas do mundo hispano-falante. Seus conhecimentos certamente serão uma contribuição que servirá para ampliar campos de interesses da revista, expandindo-a a novas regiões e especialidades.

O Conselho Editorial, como se pode comprovar numa rápida consulta à página web da revista, foi renovado, razão pela qual queremos aproveitar para expressar o nosso agradecimento não só a todos aqueles colegas que colaboraram até então com JLG como também àqueles que continuarão colaborando com ela, assim como queremos dar boas-vindas aos novos colaboradores. Queremos, ainda, estender o nosso agradecimento a todos os autores e aos que tem atuado como revisores da revista durante os seus primeiros anos de existência, aguardando pelos próximos anos com o maior dos interesses.

Quanto ao mais, continuaremos aceitando propostas de todas as áreas da geografia linguística, particularmente daquelas áreas interessadas nos avanços nas interpretações geográficas da diversidade linguística e nas questões tipológicas. 